Revue internationale P.M.E.

Économie et gestion de la petite et moyenne entreprise

\title{
La vision stratégique en contexte de PME : cadre théorique et étude empirique
}

\section{Jean-Bernard Carrière}

Volume 3, numéro 3-4, 1990

URI : https://id.erudit.org/iderudit/1007983ar

DOI : https://doi.org/10.7202/1007983ar

Aller au sommaire du numéro

Éditeur(s)

Presses de l’Université du Québec

ISSN

0776-5436 (imprimé)

1918-9699 (numérique)

Découvrir la revue

Citer cet article

Carrière, J.-B. (1990). La vision stratégique en contexte de PME : cadre théorique et étude empirique. Revue internationale P.M.E., 3(3-4), 301-325. https://doi.org/10.7202/1007983ar
Résumé de l'article

La vision stratégique est devenue un sujet d'importance au management stratégique. Dans sa partie théorique, cet article tente d'identifier la raison d'être de l'émergence de ce concept dans le champ de la stratégie ainsi que de proposer un cadre théorique en vue de son étude empirique. La partie empirique constitue une opérationalisation du concept de division, selon une perspective stratégique; en termes d'intentions, d'actions et d'impacts stratégiques spécifiques à une entreprise, tel que perçus par des décideurs de PME. Les données empiriques ont été obtenues par entrevues sur le terrain auprès d'un échantillon de 30 propriétaires dirigeants. Les résultats montrent comment la vision peut être utilisée comme outil de prospective dans l'analyse des niches au sein d'un même secteur industriel.
Tous droits réservés @ Presses de l’Université du Québec, 1990
Ce document est protégé par la loi sur le droit d'auteur. L'utilisation des services d’Érudit (y compris la reproduction) est assujettie à sa politique d'utilisation que vous pouvez consulter en ligne.

https://apropos.erudit.org/fr/usagers/politique-dutilisation/ 


\title{
La vision stratégique en contexte de PME : cadre théorique et étude empirique
}

\author{
"Nos souhaits sont les pressentiments des capacités dont nous \\ disposons, les prodromes de ce que nous serons capables d'accomplir. \\ Ce que nous désirons et $c e$ que nous pouvons, notre imagination le \\ projette hors de nous dans l'avenir. Nous ressentons la \\ nostalgie de ce que nous possédons déjà sans le savoir." \\ Goethe (autobiographie) \\ Jean-Bernard CARRIERE* \\ GREPME \\ Université du Québec à Trois-Rivières
}

\begin{abstract}
RÉSUME
La vision stratégique est devenue un sujet d'importance au management stratégique. Dans sa partie théorique, œet article tente d'identifier la raison d'être de l'émergence de ce concept dans le champ de la stratégie ainsi que de proposer un cadre théorique en vue de son étude empirique. La partie empirique constitue une opérationalisation du concept de division, selon une perspective stratégique; en termes d'intentions, d'actions et d'impacts stratégiques spécifiques à une entreprise, tel que perçus par des décideurs de PME. Les données empiriques ont été obtenues par entrevues sur le terrain auprès d'un échantillon de 30 propriétaires dirigeants. Les résultats montrent comment la vision peut être utilisée comme outil de prospective dans l'analyse des niches au sein d'un même secteur industriel.
\end{abstract}

* Jean-Bernard Carrière est professeur agrégé en management au Département d'administration et d'économique de l'Université du Québec à Trois-Rivières. Il est détenteur d'un doctorat en management (Ph.D.) de l'Université Laval. Il est également détenteur d'un MBA de l'Université du Québec à Montréal ainsi que d'une maîtrise en sciences (M.Sc.) de l'Université Dalhousie. Ses principales publications et ses intérêts de recherche portent sur la décision stratégique, le management stratégique, l'innovation technologique et l'entrepreneuriat. Adresse: GREPME, UQTR, Case Postale 500, Trois-Rivières, Québec, G9A 5 H7. 


\begin{abstract}
Strategic vision has become a significant issue in strategic management. In its theoretical section, this article suggests a reason for the emergence of this concept in the field of strategy, as well, it describes a theoretical construct to support empirical analysis. The empirical section consists in an operationalisation of the concept of vision from a strategic perspective : in terms of the intentions, actions and strategic impacts specific to a firm, as perceived by the decision makers. The empirical date analysed are from 30 field interviews with SME owners. The results show that vision can be used as a prospective tool in niche analysis within an industrial sector. Also significant statistical differences are found between performance levels and future vision for the firm. Suggestions are presented in order to use the strategic vision concept as a practical and analytical tool in strategic management.
\end{abstract}

\title{
RESUMEN
}

Ultimanente la vision estrategica ha pasado a ser un importante objeto de estudio dentro de la gestion estrategica. En la parte teorica del articulo se trata de identificar el porque de la aparicion de este concepto dentro del campo de la estrategia y tambien se trata de proponer el marco teorico que nos permita realizar un estudio de caracter empirico. La parte empirica del articulo tiene por objeto hacer operativo el concepto de vision segun una perspectiva estrategica, en base a las intenciones, acciones e impactos estrategicos especificos para una empresa tomando como punto de vista el de los responsables de las decisiones. Los datos empiricas han sido obtenidos a traves de un trabajo de campo por medio de encuestas sobre una muestra de 30 empresarios. Los resultados de la encuesta muestran como la vision puede ser utilizada como herramienta de prospectiva dentro del analisis de los nichos para un determinado sector industrial. 


\section{Introduction}

Présentement, une des questions importantes dans le domaine de la recherche en stratégie est de savoir s'il est nécessaire de connaître la vision du décideur en plus de connaître ses actions pour bien saisir le sens stratégique de ses décisions (Bennis, 1982; Bennis et Nanus, 1985; Gupta, 1984; Mendell et Gerjuoy, 1984; Westley et Mintzberg, 1989). Cet article est une exploration théorique et empirique qui s'inscrit dans cette nouvelle voie de recherche en stratégie d'entreprise, dont voici l'ordre des sections analysées :

1- Cadre théorique de la vision stratégique

2- Vision stratégique des décideurs : niveau sectoriel

3- Vision stratégique des décideurs : niveau entreprise

4- Relation entre vision et niche stratégique;

5- Relation entre vision stratégique future et actions stratégiques passées

6- Conclusion

\section{Cadre théorique de la vision stratégique}

D'entrée de jeu, la question soulevée est celle d'expliquer l'émergence du concept de vision dans le domaine de la stratégie. Du point de vue du théoricien, ce concept s'avère utile pour comprendre les processus psychologiques des décideurs d'entreprise avec l'avantage de pouvoir situer ces processus dans le contexte de la vie de l'entreprise. En retour, il permet de mieux comprendre l'influence de ces facteurs sur ces processus psychologiques, à savoir comment ces décideurs ordonnent leur univers d'entreprise en fonction de l'avenir, comment ils se proposent de le conquérir, comment ils développent une confiance pour le conquérir et, finalement, comment ils traduisent ces perceptions abstraites en termes concrets de catégories quotidiennes d'entreprises. Ce concept permet d'identifier les structures mentales du décideur ainsi que celles des membres de son entreprise. Pour ces derniers, ces structures représentent les moyens qu'ils ont en commun pour appréhender et communiquer leur réalité et leurs possibilités futures d'entreprise.

Ceci nous renvoie à d'autres questions : pourquoi est-il apparu nécessaire d'étendre le champ de la stratégie à ces nouvelles préoccupations d'analyse et pourquoi nos concepts actuels se sont-ils avérés insuffisants pour ce faire ? Une réponse plausible serait qu'un phénomène fondamental de nos sociétés établit une forte dichotomie entre, d'une part, la connaissance scientifique régie par des règles logiques, un savoir caractérisé par sa cohérence interne et sujet à vérification empirique et, d'autre part, la connaissance expérientielle puisée et vérifiée dans l'action quotidienne (Moscovici, 1981). Le concept de vision offre au chercheur un outil analytique qui lui permet d'explorer de façon théorique cette connaissance expérientielle ainsi que ses impacts sur le comportement du décideur d'entreprise. Il apparaît moins réductif que les concepts de motivations, d'attitudes et d'attributs, 
compte tenu de l'envergure de la problématique stratégique dans l'entreprise. En fait, le concept de vision permet de décrire l'ensemble du fonctionnalisme du décideur et de son entreprise, précisément celui qui orientera ses motivations, ses attitudes et ses attributs futurs. En ce sens, il s'avère utile pour mieux connaitre les contenus stratégiques futuristes du décideur, mais également pour mieux connaître et recenser les individus et groupes qui partagent ces contenus dans l'entreprise, ainsi que ceux qui les partage au sein du secteur industriel en cause, comme nous le décrivons dans la partie empirique qui suit.

\subsection{Définition de la vision}

Nous concevons la vision stratégique dans sa perspective dynamique, où elle serait plus qu'un état mental à un temps donné. Nous définissons la vision stratégique comme la dynamique de construction mentale d'un futur souhaité et possible pour une entreprise. Une théorie de la vision nous renvoie à une théorie de l'imagination en contexte d'entreprise. L'imagination devient ici une faculté qui peut avoir diverses applications à divers niveaux de «crédibilité», telle la fantaisie pour le non crédible, la vision stratégique pour le crédible. Tout comme l'imagination, la vision peut être exprimée de diverses façons, soit par des dires, des écrits ou des actions. Pour le décideur, elle constitue un outil d'exploration et de découverte qui permet de réfléchir hors de l'état actuel de ses activités pour atteindre l'état des possibles de son entreprise : d'une réflexion orientée non pas sur la bonne solution à un problème connu, mais sur la solution la plus souhaitable et possible pour un avenir encore inconnu. Ce qui distingue la vision de l'imagination fantaisiste est précisément cette relation voulue entre l'imaginable et le possible chez le décideur où, à la limite, l'imaginabilité d'un état futur devient une condition nécessaire d'acceptation de cet état comme possible.

Par contre, la vision, même lorsque conçue comme une forme d'imagination, ne sous-entend pas la production d' «images» mentales pour autant. La vision stratégique doit être conçue dans son sens métaphorique alors qu'elle a pour but de décrire la réflexion et l'imagination du décideur, bien que, comme le souligne White (1990), il y ait un certain «naturel» à croire que ce qui est imaginable a obligatoirement un fondement sensoriel et est donc «imageable». Ceci peut être attribuable à l'importance accordée à nos sens comme voie d'accès à l'imagination et particulièrement à l'importance historique de celui de la vision (sens physiologique). Le décideur n'a pas seulement les perceptions à fondement sensoriel comme sources d'imaginaire. Il peut imaginer des paradoxes, des différences entre des idées, voire même des «images mentales» sans référent perceptuel. Ce serait même restreindre la portée de l'imagination et de la vision stratégique que de la limiter à ce qui a un fondement sensoriel, à ce qui est imageable. 


\subsection{Modèle théorique d'interprétation de la vision stratégique}

Le modèle repose sur deux postulats. Un premier assume l'existence, chez le décideur, d'un lien mental entre son expérience et sa vision, c'est-à-dire l'existence d'un processus dynamique d'élaboration de schémas mentaux subséquemment entreposés dans sa mémoire et recouvrables de façon itérative dans la vision du décideur («patterns», Simon, 1986b, p. 61). Un deuxième porte sur une caractéristique évidente, bien que fondamentale à la nature humaine, soit «que les humains ont tendance à remarquer ce qui est dans leur intérêt de remarquer» (Seldon, 1984, Kirzner, 1984, p. 56). Bien que la notion de vision puisse être conçue à divers niveaux, nous nous limiterons à en préciser trois : la vision parcellaire, la vision intermédiaire et la vision générale du décideur. De ces trois niveaux, les postulats précédents nous amènent toutefois à privilégier le niveau de vision intermédiaire, là où se forment les structures mentales les plus directement identifiables aux préoccupations et décisions de niveau organisationnel dans l'entreprise. (figure 1, p. 306)

Au premier niveau de la dynamique visionnaire, se situe la vision générale du décideur, un Weltanschauung particulier au sein duquel l'on retrouve ses valeurs, ses principaux champs d'incertitude métaphysique, ainsi que les principaux axes d'intentionnalité qui l'animent. Du point de vue organisationnel, on retrouve à ce niveau-ci les intentionnalités générales et insatiables du décideur, celles qui confèrent un style spécifique à son organisation (ex. croissance).

Ce serait donc que dès ce niveau la vision est contrainte psychologiquement par les croyances et valeurs du décideur qui joueraient ici deux rôles essentiels dans la dynamique visionnaire : «Un est de simplifier : de traduire un monde qui peut être extrêmement complexe et ambigu en des termes compréhensibles et familiers. L'autre est d'assurer une continuité et une stabilité lorsque le changement menace de miner les leçons de l'expérience» (Donaldson et Lorsch, 1983, p.79-80).

A l'autre extrême de cette dynamique, l'on retrouve la vision parcellaire.: un niveau de vision où sont perçus les éléments et les changements individuels de l'environnement et de l'entreprise qui intéressent le décideur. C'est à partir de ces éléments que seront construits les schémas mentaux qui constituent la vision intermédiaire, schémas qui seront ultérieurement ajustés et intégrés aux éléments de la vision générale. A titre d'illustration, la vision de l'évolution de la situation d'approvisionnement en matière première représente une parcelle qui pourrait contribuer à la construction d'une structure mentale éventuelle, laquelle pourrait inclure également un nombre restreint d'autres parcelles touchant les concurrents, les clients et autres (Quinn 1982, Hambrick et Finkelstein, 1987).

De ces deux niveaux, $s$ 'articule une dynamique de vision intermédiaire qui porte, dans notre contexte, spécifiquement sur l'entreprise et qui correspond à la vision stratégique du décideur. A ce niveau-ci, se forment et se reforment, à partir des éléments des niveaux précédents, les schémas mentaux stratégiques les plus directement identifiables aux décisions organisationnelles. Chaque schéma mental constitue 
Figure 1

NIVEAU GENERAL

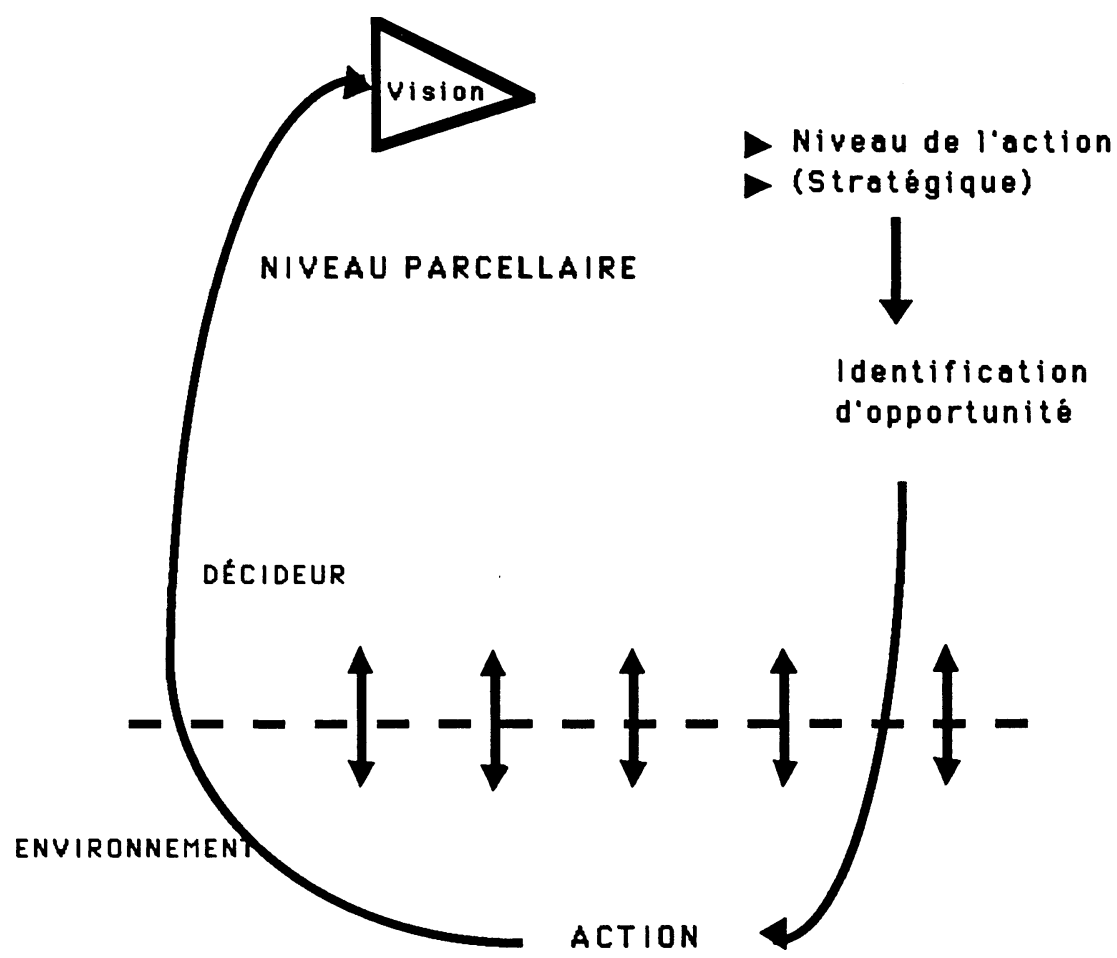

une vision d'action pour le décideur en ce que ce schéma détermine ce qui est souhaitable et possible pour son organisation à un temps donné : il constitue la vision à l'intérieur de laquelle pourront être identifiés les problèmes spécifiques, les solutions précises et les opportunités uniques pour son entreprise. Cette vision d'action se veut un cadre flexible qui tienne également compte de certaines incertitudes environnementales et organisationnelles particulièrement importantes (et donc) retenues par le décideur (Quinn, 1982). Ici la flexibilité tient largement la place de la planification en ce qu'elle laisse libre champ à la découverte alors que la planification détaillée se limite au champ de ce qui a déjà été découvert. C'est cette vision qui guide la recherche d'opportunité. Selon le modèle, l'opportunité est ici construite mentalement par le décideur; dans ce sens, nous n'assumons pas qu'elle pré-existe dans des interstices environnementaux ou organisationnels particuliers. L'opportunité constitue ici une vision particulière composée d'éléments uniques, à 
un temps donné. Par définition, cette vision n'a de valeur que pour celui qui la perçoit; et cette valeur persistera seulement aussi longtemps que les autres décideurs ne lui attacheront pas de valeur stratégique, ou encore aussi longtemps que les conditions qui la soutiennent ne changeront pas (Kirzner, 1984). C'est d'ailleurs ce qui explique que les opportunités peuvent apparaître surprenantes à ces autres décideurs : «il n'y a pas de surprise sans attente, ni d'attentes sans connaissances» (Simon, 1986, p. 11)

Comment cette vision évolue-t-elle ? Comment le décideur est-il en mesure de renouveler son cadre de vision suite à des changements souvent rapides des événements ? En partie par l'expérience acquise sous forme d'un répertoire de structures mentales déjà existantes qui lui permettent de s'adapter rapidement et intuitivement (Simon, 1986a); également par le caractère fouillé de ses observations sur divers aspects de son entreprise et de son environnement. Ceci montre que la dynamique visionnaire organisationnelle doit être continuellement alimentée et que ceci avantage les décideurs qui peuventétablir des sources structurées d'informations. A ce titre, il est à remarquer que les décideurs de notre échantillon accordent en général beaucoup d'importance et de loyauté aux réseaux d'informations qu'ils ont tissés au fil des années avec divers individus et groupes de leur secteur, notamment les clients-grossistes et les fournisseurs étrangers.

Selon le modèle proposé, la vision joue un double rôle dans l'action. Si l'expérience et le changement sont des facteurs qui déterminent l'évolution de la vision, en retour la vision oriente la perception de ces changements et l'accumulation de ces expériences. Ce serait son premier rôle. Un deuxième rôle est que la vision constitue la source d'énergie mentale nécessaire à l'action. Alors elle est non seulement une représentation mentale qui oriente l'action à entreprendre, mais aussi sa cause en ce qu'elle génère l'énergie mentale suffisante pour agir en vue de l'atteindre (Searle, 1984).

L'évolution de cette vision n'est pas toujours uniforme dans le temps. Elle peut évoluer par étapes, par sauts (Simon, 1986) d'une gestalt à l'autre selon des horizons précis, telle une année budgétaire ou une période de recouvrement. Elle peut également évoluer en envergure, selon les expériences du dirigeant.

Selon la conception de la dynamique visionnaire proposée, vision et action stratégique sont indissociables, sauf pour fins analytiques. Ce serait une dynamique par laquelle les visions d'un temps guident le décideur vers des actions futures spécifiques et individualisantes pour lui et son entreprise. Ces actions contribueront en retour à la formation des visions futures du décideur. Dans cette dynamique, vision et action stratégiques sont complémentaires. Si tel est le cas, il y a alors lieu de devoir tenir compte de ces deux processus dans l'explication du comportement stratégique du décideur et notamment sur le degré de prédiction possible de ses actions futures. La connaissance de la vision stratégique du décideur peut alors contribuer à une meilleure prédiction de ses comportements stratégiques futurs. De plus, l'agrégation de visions stratégiques individuelles pour un secteur industriel particulier permet alors au chercheur de mieux prédire l'évolution future de ce secteur. 
Pour fins d'application empirique de ce modèle, nous analysons des données obtenues lors d'une étude de terrain, portant sur l'analyse du processus de décision stratégique d'innovation de procédé dans la PME manufacturière et sa relation avec la performance (Carrière, 1990). Une partie des entrevues auprès de 30 propriétairesdirigeants d'entreprises innovatrices du secteur des scieries portait spécifiquement sur les perceptions de ces derniers quant à l) l'évolution de leur secteur et 2) de leur propre entreprise au sein de ce secteur, pour les cinq prochaines années.

Il nous est apparu nécessaire de structurer l'analyse de ces visions en des termes stratégiques concrets, selon des catégories perceptuelles et temporelles qui peuvent être mises en relation avec des actions et impacts spécifiques à l'entreprise ainsi qu'à son secteur industriel.

\section{Vision stratégique des décideurs}

Les résultats qui suivent doivent être interprétésà l'intérieur de la perspective suivante qu'ont les décideurs quant à leurs objectifs stratégiques. Sur une échelle de 1 à 5 , deux objectifs sont apparus significativement importants selon l'ordre suivant. L'augmentation de rentabilité, avec une moyenne de réponses de $4,3 / 5$, a été jugée importante ou très importante $(4,5)$ par $86,7 \%$ des répondants et l'augmentation de la croissance, avec une moyenne de 3,2/5 a été jugée importante et très importante par $36,7 \%$ des répondants. Nous n'avons pas observé de relation significative entre ces deux objectifs et les niveaux de performance des entreprises. Quant aux autres objectifs de divers ordres, ils sont apparus importants ou très importants $(4,5)$ pour seulement $14,7 \%$ des décideurs répondants. De façon générale, rentabilité et croissance sont perçues par ces décideurs comme formant un équilibre où la survie organisationnelle prime et doit être assurée par une certaine rentabilité, mais où il y a également «un certain naturel à vouloir croître», au rythme des possibilités qu'offrent le secteur et l'entreprise. Ainsi, même si certains considèrent que leur entreprise a atteint une taille optimale, ils appréhendent que sans le momentum qu'apporte une certaine recherche de croisance, il est plus difficile pour l'équipe de traverser les situations difficiles et qu'alors «l'entreprise meurt naturellement». C'est donc dansce contexte que les décideurs cherchent à développer des avenues de survie et d'expansion propices à leur entreprise. Les analyses qui suivent ont pour but de décrire quelles sont les visions stratégiques que ces décideurs ont développées pour atteindre les objectifs généraux.

Les résultats d'ensemble montrent que trois principales dimensions de vision émergent comme autant de thèmes dominants dans l'esprit des décideurs pour les prochaines années au sein de l'échantillon. Ces dimensions sont récurrentes au niveau de la vision sectorielle ainsi qu'au niveau de la vision organisationnelle des décideurs. Elles portent essentiellement sur :

1) les possibilités d'approvisionnement futurs en matière première (ressource forestière); 


\section{Tab. 1 Vision de l'évolution du secteur}

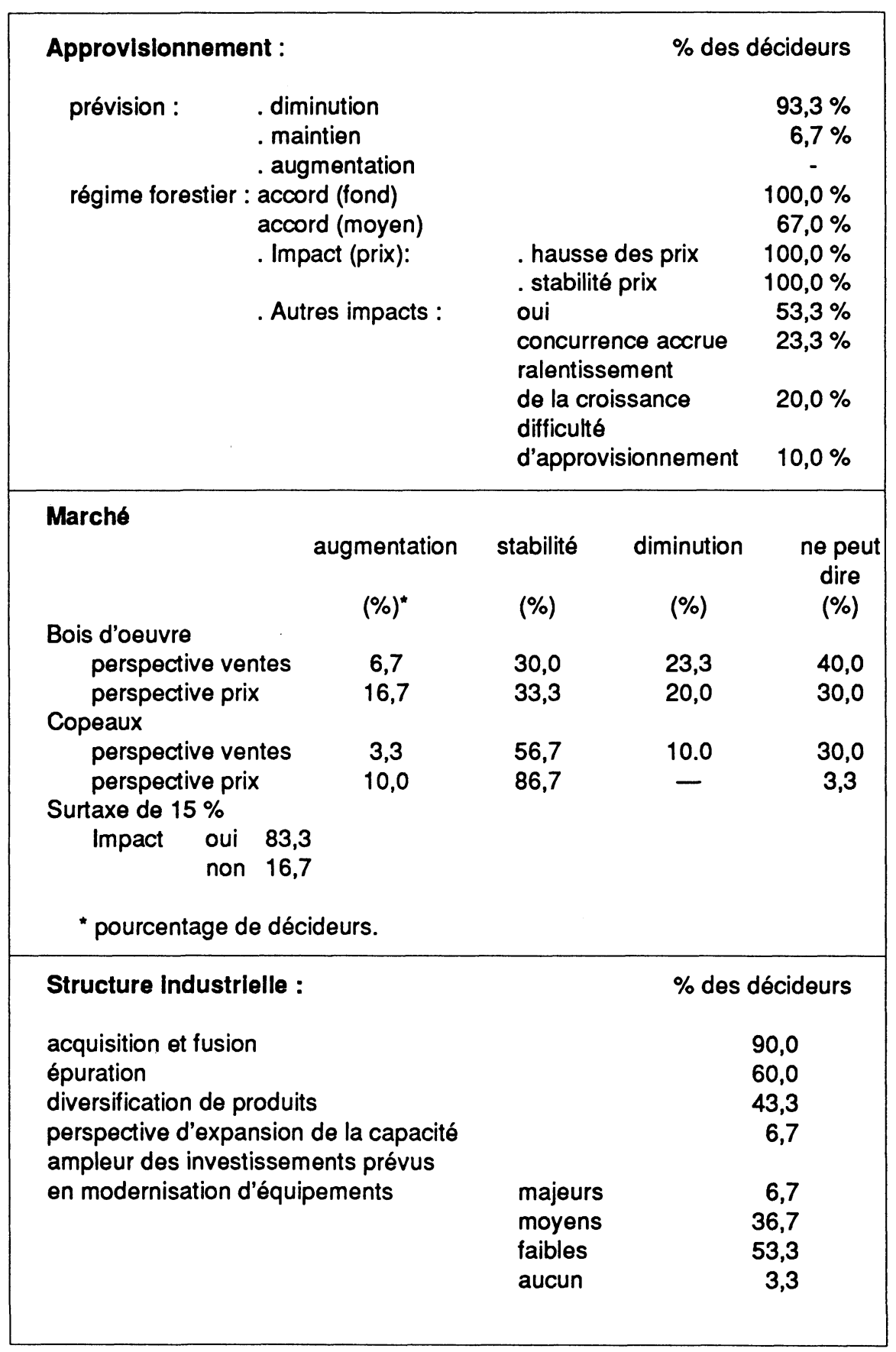


2) les perspectives futures du marché du bois d'oeuvre;

3) la restructuration éventuelle de ce secteur industriel.

L'analyse des résultats se concentre sur l'approfondissement de chacune de ces trois dimensions stratégiques et ce, à deux niveaux d'analyse, soit la vision de niveau sectoriel (tableau 1) ainsi que la vision de niveau organisationnel (tableau 2).

\subsection{Dimension approvisionnement}

Les résultats de niveau sectoriel sur la dimension approvisionnement montrent que la vision des décideurs porte surtout sur la prévision possible des approvisionnements totaux pour le secteur (tableau 1, p. 309).

Les décideurs envisagent de façon presque unanime $(93,3 \%)$ une diminution des approvisionnements au cours des prochaines années, conséquence de la surexploitation des années antérieures. L'autre principale préoccupation consiste à anticiper les impacts encore incertains d'une nouvelle politique gouvernementale de conservation de la ressource forestière présentement en voie d'implantation. Ce nouveau régime forestier impose des plafonds et, même pour certaines régions des réductions dans les allocations forestières, ainsi que de nouvelles exigences de reboisement pour les entreprises de ce secteur. Notons que les décideurs sont tous d'accord ( $100 \%$ ) avec le principe de fond de ce nouveau régime forestier qui fixe le taux d'exploitation de la forêtà un niveau qui assure un rendement soutenable à long terme. Les décideurs sont également en accord, pour les deux tiers d'entre eux, avec les moyens établis pour atteindre cet objectif. En somme, il leur apparaît qu'il était plus que temps d'agir, af in de corriger ce qu'ils perçoivent comme une surexploitation destructrice à long terme de la forêt et, par intérêt, délétère à leur secteur d'activité. Toutefois, le tiers $(33,3 \%)$ des répondants n'est pas d'accord avec les moyens annoncés pour y parvenir. Ces désaccords portent surtout, pour $20 \%$ de ces répondants, sur un rythme d'implantation du régime jugé trop rapide, compte tenu de la période d'adaptation qu'ils estiment nécessaire pour s'ajuster à des coûts de fabrication plus élevés. Pour d'autres (13,3\%), ce sont plutôt les incertitudes reliées à la nature et l'envergure de ces coûts qui pose problème.

En dépit de certaines réticences de niveau opérationnel, tous les décideurs (100\%) partagent la même vision quant aux avantages majeurs au niveau sectoriel; une plus grande stabilité de leurs approvisionnements conséquenteà un plafonnement de l'offre future pour ces derniers, ainsi qu'une hausse éventuelle des prix pour leurs produits explique ce consensus quant au fond. Les décideurs sont apparus très sensibles à ces impacts, et à l'aise dans la manipulation mentale de liens possibles entre la création de cette rareté et la création de profits supplémentaires pour leur secteur. Selon ces résultats, il est clair que le consensus des décideurs sur ces deux impacts témoigne d'une aire de vision pour laquelle ils entretiennent très peu d'incertitude. En somme, une plus grande stabilité d'approvisionnements ainsi qu'une hausse éventuelle des prix constituent deux «constantes» inévitables sur 
lesquelles ils peuvent miser en vue d'articuler leurs stratégies futures. Une des raisons de cette certitude est leur perception que le ministre responsable de ce secteur a élaboré une politique d'allocation dans laquelle il s'est laissé relativement peu de marge discrétionnaire, contrairement aux années antérieures où l'intervention politique pouvait permettre d'obtenir des allocations additionnelles. Ainsi, ils voient que non seulement le nombre d'allocations «politiques» sera diminué, mais également que chaque allocation devra être davantage justifiée comme étant équitable envers les autres scieries. A ce propos, ils ont confié un mandat de vigilance à leur association sectorielle.

Tab. 2 Vision de l'évolution de l'entreprise individuelle

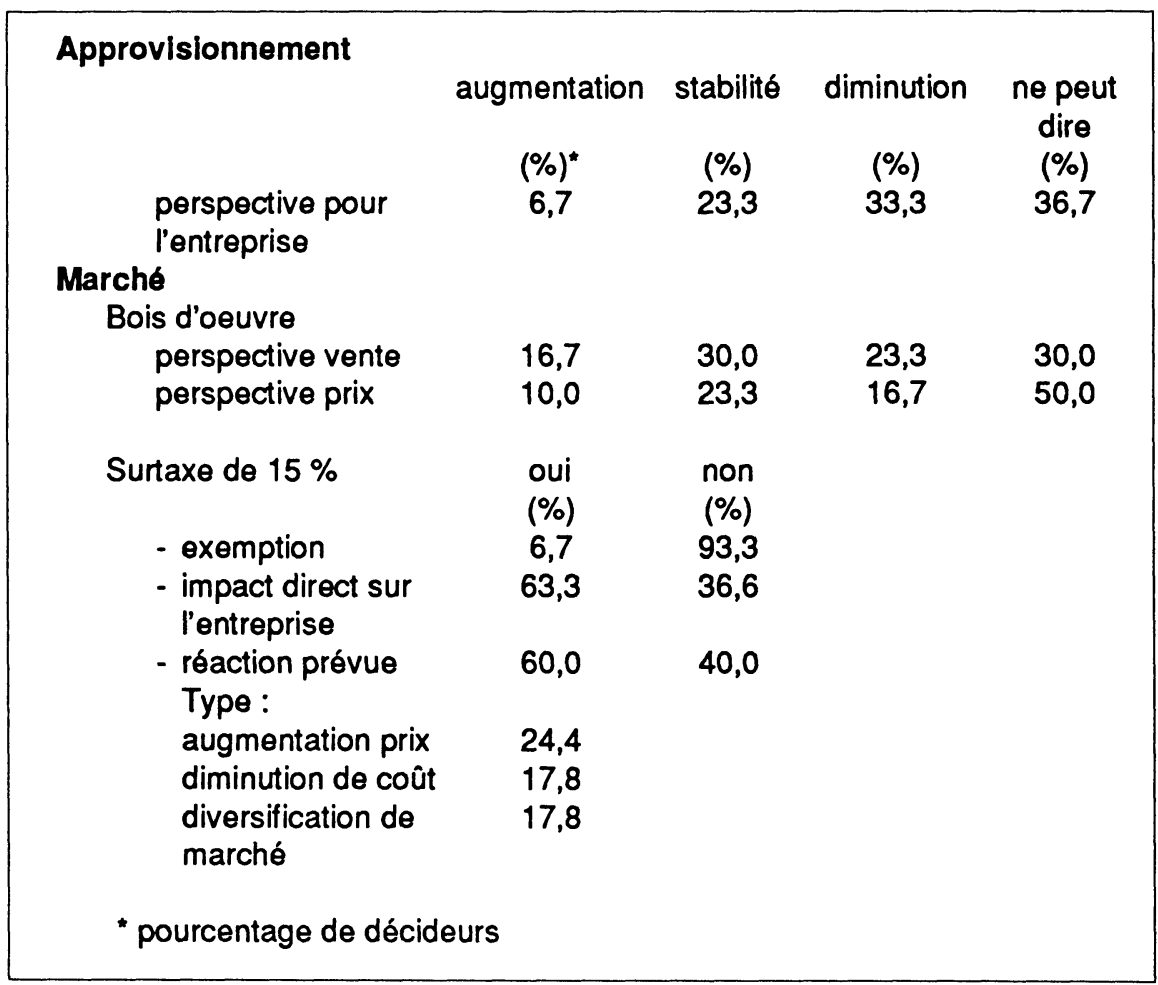

Toutefois, plus de la moitié des décideurs de l'échantillon voient également d'autres impacts à caractère plus négatif 53,3\% (tableau 1, p. 309). Ainsi, 23,3\% des décideurs voient une concurrence future accrue sur les approvisionnements, $20 \%$ entrevoient un impact négatif sur la croissance de leur secteur et $10 \%$ entrevoient que certaines usines du secteur auront de sérieux problèmes de survie attribuables à des réductions d'approvisionnement. Par contre, seulement 33,3\% des décideurs prévoient avec certitude une réduction d'allocation pour leur propre entreprise (tableau 2). De plus, deux tiers des décideurs entrevoient même pouvoir maintenir leur propre 
approvisionnement à leur niveau actuel $(23,3 \%)$, voire de l'augmenter $(6,7 \%)$, dans ce contexte de rareté future. Pour $36,7 \%$ d'entre eux, le problème d'approvisionnement demeure encore très nébuleux, encore ouvert à diverses avenues. En somme, les écarts dans les résultats entre ces deux niveaux d'analyse de la vision stratégique sont révélateurs du degré de confiance et de la vision de contrôle qu'entretiennent les décideurs innovateurs de ce secteur quantà leur propre capacité stratégique de réussir à se négocier une niche d'approvisionnement préférentielle pour l'avenir, cette vision étant fondée sur leur profonde expérience du secteur. La durée moyenne d'expérience de gestion des décideurs au sein de leur secteur est de 15.7 ans.

\subsection{Dimension marché}

La deuxième principale dimension de vision sectorielle porte sur les perspectives de marché futur. Les résultats montrent qu'à l'intérieur de cette vision, les décideurs entrevoient des perspectives distinctes pour le marché du bois d'oeuvre et pour celui des copeaux (tableau 1, p. 309).

\subsubsection{Marché du bois d'oeuvre}

Pour le marché du bois d'oeuvre, $36,7 \%$ des décideurs anticipent un niveau sectoriel de vente stable ou même croissant pour l'avenir; $23,3 \%$ prévoient une diminution des ventes et $40 \%$ ne peuvent concevoir de perspectives précises de vente pour les prochaines années. Il en va de même pour les perspectives futures des prix du bois d'oeuvre, bien que les visions sur ce point apparaissent légèrement plus optimistes et plus certaines (30\% incertitude/prix par rapport à $40 \%$ incertitude/ vente). Cette différence de résultats s'explique par la plus grande rareté des futurs approvisionnements pour ces entreprises, ce qui aurait pour effet de créer un seuil relativement solide pour les prix du bois d'oeuvre. Les résultats montrent que sur cette dimension marché, les visions des décideurs sont surtout orientées vers la prévision de changements dans les prix plutôt que dans les volumes de ventes. Ceci est attribuable à ce que, dans l'esprit des décideurs, le marché du bois d'ouvre est un vaste marché, anonyme mais toujours ouvert à un certain prix. Au-delà de 6 mois, ils admettent ne pouvoir prévoir avec certitude le moment exact et l'ampleur du changement futur dans le marché. C'est à partir de cette limite qu'ils recherchent activement de l'information de marché qui pourrait leur donner des indices sur le nombre et le \% de baisse des prix. Aussitôt qu'ils peuvent percevoir ces prochains niveaux planchers, ils sont alors en mesure de traduire mentalement ces seuils en cible de réduction de coût et ce, avant que les prix ne tombent à ces niveaux. De cette façon, ils estiment pouvoir traverser la baisse de marché anticipée tout en maintenant leurs opérations. Le tableau 2, p. 311, montre que, à l'opposé de ce qui était le cas pour la dimension approvisionnement, ce que les décideurs perçoivent icicomme conditions futures de marché pour leur secteur est également ce qu'ils entrevoient au niveau de leur propre entreprise, bien qu'avec moins d'incertitudes quant à leurs propres ventes 
futures et plus d'incertitudes quant aux prix qui pourraient être obtenus pour leurs propres produits. En somme, étant donné la nature anonyme et ouverte du marché du bois d'oeuvre, différent du marché plus personnalisé de l'approvisionnement en matière première, les décideurs ne perçoivent pas pouvoir performer plus efficacement que leurs concurrents sur ce marché.

\subsubsection{Marché des copeaux}

La vision des décideurs quant à l'évolution du marché des copeaux est tout à fait différente de celle qu'ils entretiennent pour le marché du bois d'oeuvre. La majorité des décideurs $(56,7 \%)$ entrevoient que la demande (volume) sera stable sur le marché des copeaux, tandis que seulement $30 \%$ entrevoient une stabilité équivalente pour le marché du bois d'oeuvre. Également, ils n'entretiennent pas ou peu d'incertitude $(3,3 \%)$ quant à l'évolution future des prix pour les copeaux, alors que la grande majorité des décideurs $(86,7 \%)$ entrevoient qu'il n'y aura pas de baisse significative des prix pour ce produit alors que seulement $33,3 \%$ d'entre eux entrevoient des conditions similaires pour leurs produits de bois d'oeuvre.

La raison de cet écart est que le marché des copeaux s'apparente à un oligopsone alors qu'il y a peu d'usines de pâtes et papiers clientes, de sorte que les augmentations de prix sont minimales et les marges bénéficiaires historiques sur ce produit extrêmement minces, voire inexistantes pour les PME sous étude. Les décideurs considèrent donc les copeaux davantage comme un sous-produit de leurs opérations. Dans ce sens, ils sont surtout intéressés à en diminuer le volume de fabrication au minimum, ce qui expliquerait que ce produit n'occupe pas une place importante dans leur cadre de vision stratégique.

Notons enfin que les décideurs estiment qu'une surtaxe de $15 \%$ àl'exportation des produits de bois d'oeuvre aux Etats-Unis, imposée par le gouvernement canadien suite à des pressions politiques américaines, a eu un impact concurrentiel significatif sur les entreprises du secteur. En effet, 83,3 \% (tableau 1, p. 309) d'entre eux entrevoient qu'elle aura un impact significatif sur leur position sectorielle de marché, bien que seulement $63,3 \%$ (tableau 2, p. 311) perçoivent que cette surtaxe aura un effet direct sur leur propre position individuelle. De ces dernières, $60 \%$ ont déjà anticipé une riposte spécifique, telle une augmentation de prix $(24,4 \%)$, des efforts de réduction de coût $(17,8 \%)$ ou encore une diversification vers les marchés outremer $(17,8 \%)$ (tableau 2) (Cette surtaxe a depuis été réduite.

\subsection{Dimension structurelle}

Dans leur troisième dimension de vision stratégique, les décideursentrevoient une restructuration importante de leur secteur pour les cinq prochaines années. Ainsi, $90 \%$ d'entre eux anticipent des acquisitions et fusions d'entreprises soit au sein du secteur, soit par des entreprises du secteur des pâtes et papiers intéressées à rehausser 
leur contrôle sur l'approvisionnement en forêt. Toutefois, seulement $43,3 \%$ entrevoient l'éventualité d'être eux-même sujet d'acquisition (tableau 3, colonnes 7 et 9). De même, $60 \%$ des décideurs anticipent des fermetures d'entreprises au sein de leur secteur bien qu'une seule entrevoit cette éventualité pour elle-même (tableau 3, colonne 10). En ce qui regarde les nouvelles avenues de croissance, $43,3 \%$ des décideurs prévoient que le secteur sera contraint de croître partiellement hors de ses gammes de produits actuels, alors que 53,3\% voient cette éventualité pour leur propre organisation (tableau 3 , colonne 3 ). Ceci suggère que cette option stratégique est essentiellement limitée à ceux qui la perçoivent possible au niveau de leur secteur. Peu de décideurs entrevoient une augmentation de capacité de fabrication au sein de leur secteur et, conséquemment, d'investissements majeurs pour la période sous étude, compte tenu du plafonnement de la ressource forestière.

\subsubsection{Conclusion}

Dans l'ensemble, ces résultats suggèrent qu'il y a lieu d'être prudent lorsqu'après avoir identifié de façon prospective un déterminant d'action au niveau sectoriel, d'assumer qu'il en sera un d'égal déterminisme pour un décideur individuel de ce secteur lors de sa prise de décision. En effet, si à un niveau agrégé on entretient une certitude à priori quant à une certaine relation de cause à effet, la diversité des situations individuelles d'entreprise constitue autant d'interstices sectoriels recelant des intentions et des incertitudes locales aptes à leur permettre de se définir de nouvelles marges de manoeuvre. Un décideur peut alors élaborer ses propres scénarios (visions) de relation de cause à effet selon sa situation particulière d'entreprise (localisation, essences exploitées, type d'utilisation, relations politiques). Ces résultats empiriques montrent de façon générale que les visions stratégiques développées par les décideurs de l'échantillon ont comme postulat sous-jacent une confiance de pouvoir contrôler l'évolution de leur approvisionnement d'entreprise et, plus globalement, de l'évolution de leur entreprise au sein de leur secteur, pour les années à venir.

Cette analyse des principales trajectoires d'évolution sectorielle telle «qu'envisionnée» par les décideurs mène à poser la question suivante : quelles sont les stratégies spécifiques conçues par ces décideurs pour survivre et prospérer dans un environnement sectoriel qu'ils décrivent comme instable et appauvri pour les prochaines années? Nous tentons de répondreà cette question dans la section qui suit.

\section{$4 \quad$ La vision stratégique individuelle des décideurs}

Pour ce faire, nous reconstruisons la vision stratégique des décideurs pour les cinq prochaines années selon dix dimensions stratégiques retenues de l'analyse des données empiriques. Ces dimensions visionnaires spécifiques à chaque décideur sont décrites au tableau 3. 
Tab. 3 Cadre de vision stratégique des décideurs individuels

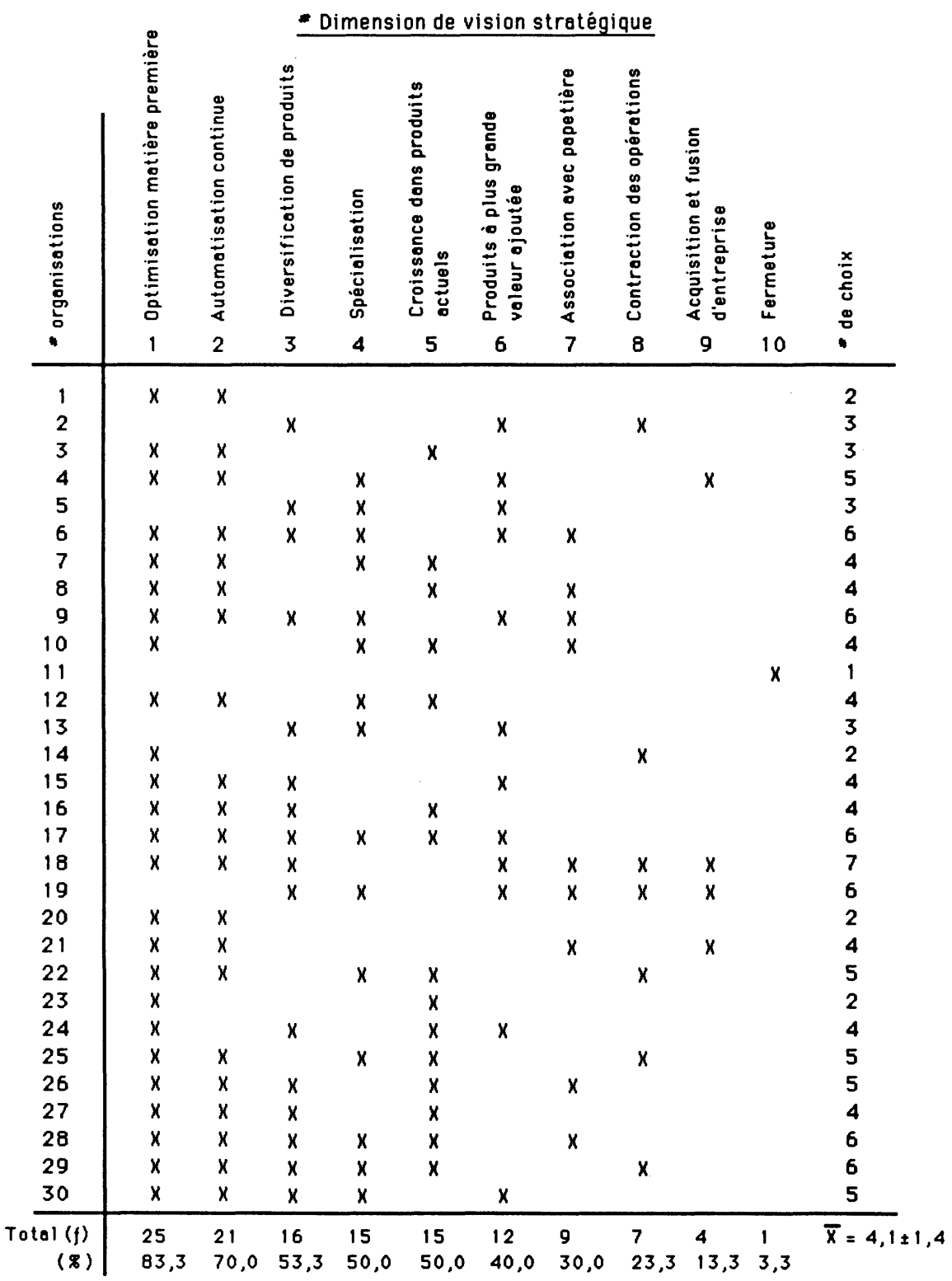


Nous observons qu'un cadre de vision stratégique est formé en moyenne de 4,1 dimensions, allant d'une dimension individuelle (entreprise \#11) à sept dimensions visionnaires pour une entreprise (\#18). Les dimensions sont inscrites de gauche à droite dans le tableau selon la fréquence totale dans l'échantillon. Ces résultats seront analysés en terme de sous-groupes de dimensions stratégiques distinctes.

\subsection{Dimensions visionnaires de rentabilité}

On note que les deux premières dimensions les plus fréquentes (1 : optimisation de la matière première et $2:$ automatisation continue) portent sur des aspects stratégiques reliés plus directement à la rentabilisation soutenue des opérations présentes plutôt qu'à un objectif de croissance. Ainsi, 83,3\% des décideurs entretiennent dans leur cadre visionnaire individuel la dimension d'une plus grande optimisation de la matière première. Pour les $16,7 \%$ des décideurs qui ne l'ont pas retenu, cela s'explique par le fait que des efforts en ce sens ont déjà été réalisés (entreprises \#2, \#5, \#13 et \#19) ou encore, qu'ils ne sont pas pertinents (entreprise \#11, fermeture). En deuxième place, la dimension «automatisation continue» est présente dans $70 \%$ des cadres visionnaires, alors que les décideurs entrevoient maintenir leurs efforts de renouvellement des équipements. Ces résultats sont révélateurs d'une approche stratégique prudente face au renouvellement technologique, et en équilibre avec des périodes de recouvrement financier (moyenne 3,2 ans) jugées appropriées par des décideurs qui se voient contraints d'opérer dans un contexte futur de non-croissance et de faible rentabilité. En ce qui regarde les $30 \%$ de décideurs qui n'ont pas jugé ces dimensions stratégiques prioritaires pour les prochaines années, ce sont ceux qui, sauf pour deux exceptions, estiment avoir déjà réalisé des investissements suffisants en nouvelles technologies ces dernières années. Les deux cas d'exceptions s'expliquent : dans une entreprise, le décideur poursuit une stratégie d'attentisme par rapport à ses concurrents alors qu'il jouit d'un avantage concurrentiel important quant à ses coûts d'approvisionnement de par sa localisation; quant à l'autre, elle entrevoit à moyen terme mettre fin à ses opérations à moyen terme.

\subsection{Dimensions visionnaires de croissance}

Six dimensions de vision stratégique portent sur des thèmes reliés à l'évolution et l'innovation de produit et sont plus directement reliées à un objectif de croissance de l'entreprise. Les quatre premières (3: diversification de produits, 4 : spécialisation, 5 : croissance dans les produits actuels et $6:$ produits à plus grande valeur ajoutée) font référence à des orientations de croissance interne. On note que $83,3 \%$ du total des visions stratégiques contiennent au moins une de ces dimensions et que près de la moitié $(47,5 \%)$ de ces visions possèdent, selon une combinaison quelconque, plus d'une dimension. Les $16,7 \%$ des décideurs qui n'ont pas retenu l'une de ces dimensions de produits sont ceux qui n'envisagent pas de croissance future pour leur entreprise, soit qu' ils choisissent de parfaire leurs activités actuelles 
(entreprises \#1, \#20 et\#21), soit qu'ils prévoient vivre une décroissance importante de leurs activités faute d'approvisionnement (entreprises \#11 et \#14).

Deux autres dimensions peuvent être davantage reliées à des orientations de croissance externe : 7 : association avec papetière et 9 : acquisition et fusion d'entreprise. L'on note que $33,3 \%$ des décideurs ont retenus une de ces deux stratégies comme voie de croissance future, alors que $10 \%$ du total des décideurs ont retenu ces deux stratégies de croissance externe. Finalement, deux dimensions visionnaires sont reliées à des orientations de décroissance : 8 : contraction des opérations et 10 : fermeture, elles ont été retenues par $26,7 \%$ des décideurs. Pour les dimensions 7 et 9 , le contraste entre les résultats sur les visions organisationnelles et ceux sur les visions sectorielles est révélateur. Alors que dans la section précédente (tableau 1), $90 \%$ des décideurs prévoient qu'il y aurait davantage d'acquisitions et de fusions au niveau de leur secteur durant les prochaines années, on observe qu'au niveau des entreprises individuelles, ce ne sont que $30 \%$ des décideurs qui envisagent une acquisition ou une fusion pour leur propre entreprise, et $13,3 \%$ qui envisagent une association éventuelle avec une papetière, pour un total de $43,3 \%$ des décideurs. Il en va de même pour les épurations possibles au sein de la population d'entreprises du secteur. Alors que $60 \%$ des décideurs prévoient une épuration probable au sein de leur secteur, seulement un $(3,3 \%)$ envisage qu'il en sera victime. Ces résultats montrent comment chaque décideur s'adresse aux grands objectifs stratégiques que sont la rentabilité et la croissance/décroissance en fonction de sa situation d'entreprise. Ils montrent que ces objectifs recèlent une gamme de visions stratégiques spécifiques voire uniques à chaque entreprise. Que ces visions peuvent être rendu accessibles à l'interprétation empirique lorsque analysées selon des combinaisons distinctes d'éléments stratégiques, ce qui permet de mettre en évidence les éléments communs à ces diverses visions stratégiques. Ces résultats suggèrent que les décideurs auraient tendance à estimer de façon favorable leur propre probabilité de survivre comme entreprise indépendante pour les années à venir, ce qui va dans le sens du postulat déjà mentionné.

\section{$5 \quad$ Vision et niche stratégique}

L'analyse précédente sur les visions individuelles des décideurs soulève deux questions que nous poursuivons dans cette section : compte tenu que les décideurs individuels perçoivent ces diverses dimensions comme un tout, une «gestalt», quelles sont les diverses niches distinctes qui peuvent être identifiées au sein du secteur ? Considérant que les décideurs auront vu à se définir une niche spécifique de façon à minimiser la concurrence au sein du secteur, comment ces niches s'agencent-elles au sein du secteur? 


\subsection{Description des niches individuelles}

Pour tenter de répondre à ces interrogations, une analyse de regroupement a été effectuée en utilisant les dimensions de vision organisationnelle selon leurs séquences de présentation au tableau 4. Les résultats montrent comment chaque décideur a pu se définir une niche stratégique relativement unique en termes des dimensions visionnaires précédentes.

Un parcours horizontal du tableau 4 nous montre la position finale des niches stratégiques (chiffres encerclés). L'on observe que les dix dimensions retenues permettent de différencier des niches individuelles pour $66,6 \%$ des entreprises de l'échantillon. Pour le 33,3\% des entreprises où les décideurs ont des niches communes, il est relativement simple de les différencier en ajoutant une dimension visionnaire additionnelle. Ceci permet de fragmenter les cinq groupes résiduels en niches individuelles distinctes (\#6 et \#9, \#16 et \#27, \#7 et \#12, \#22 et \#25, \#5 et \#13). C'est ce que nous nous proposons de décrire dans les paragraphes qui suivent.

\subsection{Justification des groupes résiduels}

La caractéristique des groupes \#6 et \#9 est le regroupement des deux décideurs possiblement les plus sensibles et aptesà la mise en marché de nouveaux produits au sein de l'échantillon. L'entreprise \#6 est en bonne situation financière, mais n'est pas rébarbative à entrer en partenariat avec une papetière, pour autant qu'elle puisse en conserver le contrôle. Par cette association, elle anticipe pouvoir jouir d'une plus grande sécurité et d'un plus grand volume d'approvisionnement, mais également d'avoir une assise financière plus solide. Ceci lui permettrait de poursuivre les pistes de diversification déjà amorçées (bois pré-coupé et traitement de préservation de ce bois). L'entreprise \#9 est de plus grande taille et la diversification constitue, dans ce cas-ci, la principale voie stratégique future. Le décideur envisage de faire évoluer son organisation de façon globale vers la transformation secondaire «quitte à acheter d'une autre entreprise la matière première qu'il me faut». Pour ce faire, certains membres-clé de l'organisation sont à élaborer, de concert avec des organismes de recherche, de nouveaux produits à partir de la fibre de bois (ciment à base de bois).

Les entreprises des groupes \#16 et \#27 recherchent de façon commune la diversification vers de nouveaux produits et la croissance par la gamme de produits actuels. Cependant, \#16 entrevoit de se diversifier davantage vers la production de bois vert pour le marché outre-mer, étant donné sa proximité d'un port de mer, alors que l'entreprise \#27, dont la principale activité est le rabotage à forfait, entrevoit se diversifier davantage dans le courtage de bois et la consultation technique.

Les entreprises \#7 et\#12 entendent poursuivre une politique d'automatisation continue, mais elles partent de seuils technologiques très différents. L'entreprise \#7 (vente : 9,2 millions) en est à réaliser des innovations de procédés techniques de pointe pour le secteur, afin d'optimiser le rendement d'une matière première d'une 


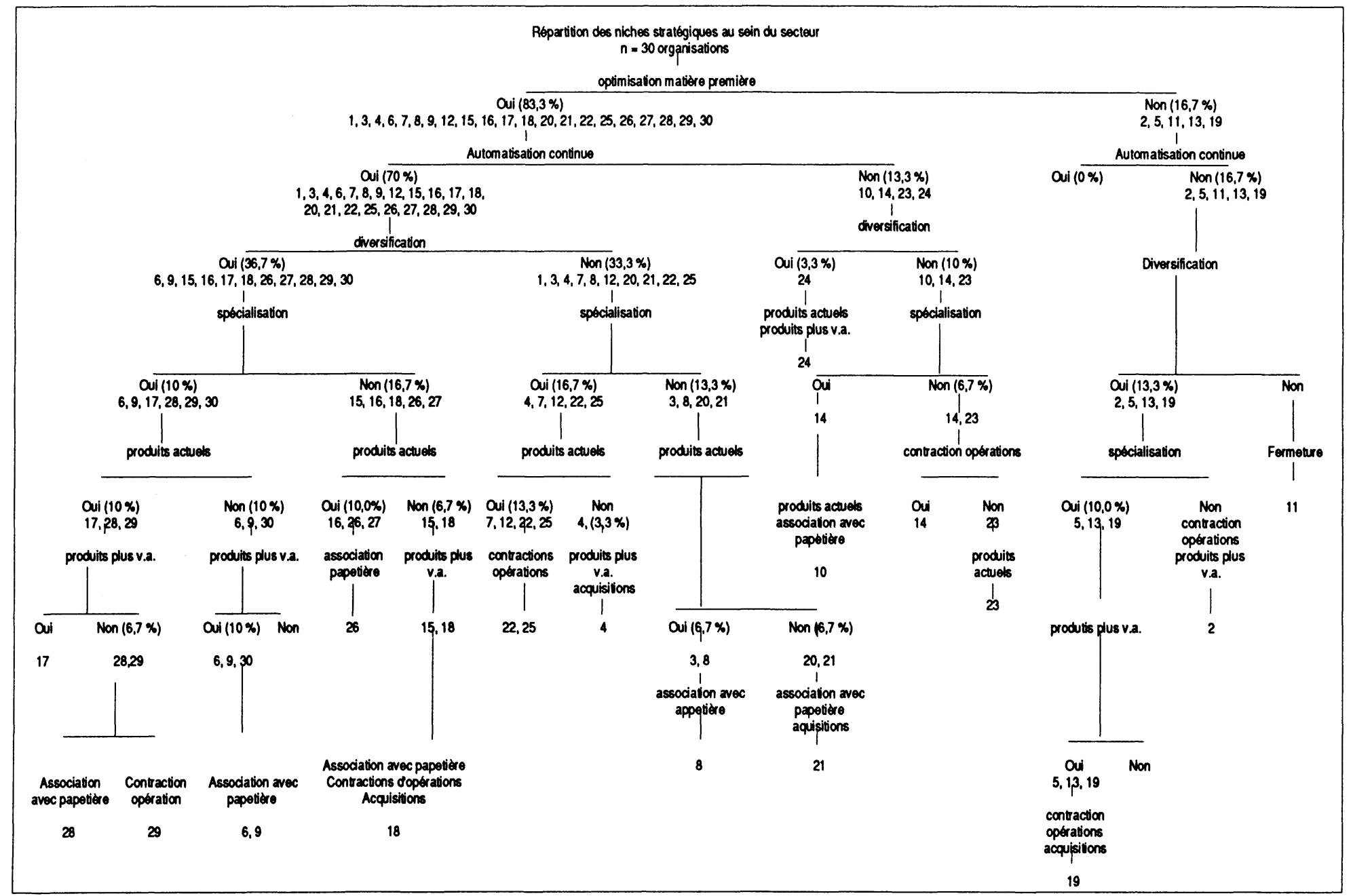


très haute qualité. L'entreprise \#12 est une organisation de plus petite envergure (vente : 1,3 million) et moins rentable (marge nette : $2,7 \%$ vs $12,8 \%$ pour le \#12). Les innovations de procédés y sont plus conventionnelles, de coût modeste et à contenu technique relativement plus simple bien que techniquement astucieuses. Dans les deux cas, on semble avoir réussi à réduire au minimum la production de copeaux. Dans le cas de \#7, cette minimisation a été réussie par l'automatisation des procédés de fabrication, ce qui a permis d'optimiser les diverses coupes de bois (17 $\%$ de la production totale en copeaux). Pour le \#12, cette minimisation a été réalisée en spécialisant le système de fabrication vers l'optimisation des coupes de petites dimensions. Pour le \#12 également, la qualité de sa matière première lui permet de produire un pourcentage significatif de grande dimension ( $2 \times 10 \times 16)$. Ces deux facteurs ont contribué à réduire la production de copeaux ( $9 \%$ de la production totale en copeaux).

Les entreprises \#22 et \#25 représentent deux cas de contraction éventuelle des opérations, faute d'approvisionnement. Elles ont en commun une décroissance qui nécessitera le délaissement de certains produits pour une spécialisation accrue dans un ou quelques-uns de leurs produits/services actuels. Mais leur spécialisation respective differe. Pour \#22, cette spécialisation portera exclusivement sur le sciage à forfait, alors que pour \#25, elle portera sur la transformation de bois de petites dimensions tels le 1 X 3 , le 1 X 4 et le 1 X 6 .

Pour les groupes \#5 et \#13, les distinctions de niches sont importantes. L'entreprise \#5 en est une de grande taille au sein du secteur (ventes : 50,5 millions). Au plan fabrication, elle a déjà investi de façon substantielle dans des technologies de pointe. Ceci lui donne un rendementet une flexibilité manufacturière «intouchable» selon son décideur. De plus, du point de vue de la mise en marché, cette entreprise a élargi sa diversification de marché, pour devenir spécialiste de l'exportation outremer. Cette diversification lui permet d'envisager une croissance à partir de ses produits actuels pour les prochaines années, bien qu'elle demeure consciente de la nécessité d'une éventuelle diversification vers des produits à plus de valeur ajoutée. L'entreprise \#13 est de plus petite taille (vente : 3,7 millions). Elle se spécialise dans la transformation d'essences de feuillus en spécialités diverses (bois de meuble précoupé, traverses), mais elle envisage une diversification future plus poussée (panneaux d'avion). Un parcours à la verticale du tableau 4 permet d'observer l'individualisation progressive des niches stratégiques, de préciser leur originalité et également d'établir divers niveaux de généralisation relative (contingentielle).

\subsection{Conclusion à la section}

A tout le moins, il apparait clair, suite à la description précédente, que des visions et des niches stratégiques uniques peuvent être identifiées pour chaque décideur et entreprise de l'échantillon. Comme l'a souligné un des décideurs : «... il n'y a pas deux scieries pareilles» (\#6). Alors, même si l'on a parfois tendance à 
considérer ce secteur industriel comme étant très homogène, cette perception ne résiste pas à un examen critique des réalités stratégiques des entreprises du secteur et particulièrement des visions du futur entretenues par les dirigeants.

Le type et le niveau d'analyse retenu pour cette étude mettent en évidence la composante entrepreneuriale (ou volontariste) de la vision d'action individuelle des décideurs et, par le fait même, d'atténuer le degré de pré-détermination des facteurs structuraux sur le comportement final des décideurs. Cette analyse montre que l'évolution d'un secteur, même en apparence homogène, est en bonne partie ouverte, qu'elle peut être conçue comme étant toujours indéterminée à un certain degré, la preuve étant la persistance de l'entrepreneuriat. Par conséquent, il apparaît en pratique impossible de prédire l'évolution d'un secteur seulement à partir de quelques facteurs sectoriels «déterminants»; ceci pour la simple raison que cette évolution est tributaire non pas de ces quelques facteurs, mais plutôt de la réaction innovatrice et imprévisible des décideurs à un ensemble spécifique de facteurs sectoriels et d'entreprises. Ceci suggère qu'une meilleure prévision de l'évolution d'un secteur pourrait être obtenue par une analyse stratégique axée sur ce que les éléments les plus dynamiques d'un secteur entrevoient et projettent réaliser pour une période future spécifique. Il apparaît ainsi possible et souhaitable d'enrichir l'analyse (microéconomique) sectorielle en la complétant par une perspective stratégique de façon à montrer l'évolution d'un secteur de façon moins réductrice et moins déterministe. En somme, il faudrait montrer comment cette évolution sectorielle est également déterminée par une diversité de visions et de décisions entrepreneuriales.

\section{Vision stratégique future et action stratégique antérieure}

La diversité des visions et niches décrites soulève une autre question, à quel degré la vision future du décideur peut-elle être influencée de façon significative par le succès de ses actions stratégiques antérieures. Le but visé ici n'est évidemment pas de mettre à l'épreuve la justesse de la vision future des décideurs à succès. Il s'agit plutôt de savoir comment ces «gagnants actuels» de l'échantillon perçoivent l'avenir de leur secteur ainsi que de leur entreprise, par comparaison avec ceux qui n'ont pas atteint le même degré de succès pour une mêmé période donnée.

A cette fin, nous avons effectué une analyse discriminante sur les divers éléments visionnaires retenus par les décideurs selon leur niveau de performance de marché pour les trois années antérieures à leur description de leur vision stratégique future. Le niveau de performance de chaque entreprise fut établi comme étant significativement au-dessus $(\mathrm{P}+)$, au-dessous $\left(\mathrm{P}^{-}\right)$, ou le même $(\mathrm{P})$ que celui de l'échantillon. Pour ce faire, les indicateurs de performance retenus furent la rentabilité (\% marge nette, $\%$ rendement sur actif) et la croissance (\% de croissance des ventes et $\%$ croissance des actifs) sur trois ans. Ceci a permis d'identifier une fonction discriminante significative $(\mathrm{p}=0,05)$ comprenant quatorze variables, à l'aide de 
laquelle $96,7 \%$ des entreprises ont pu être correctement classifiées. Les variables ont été regroupés sous forme de profils de performance qui sont montrés au tableau 5.

Tab. 5 Relation entre la vision stratégique future et la performance antérieure

P-

$$
\text { P+ }
$$

\section{VISION FUTURE DU SECTEUR}

\section{Structure}

- Recherche produit à plus de valeur ajoutée

- Intégration aux papetières

- Fusion et acquisition

\section{Marché}

- Perspective prix du bois

- Régime forestier; conséquences

\section{Approvisionnement}

et autres impacts

\section{VISION FUTURE DE L'ENTREPRISE}

Marché

- Perspectives de ventes

- Surtaxe de $15 \%$

- Perspectives organisationnelles

\section{Approvisionnement}

- Contraction des opérations

- Automatisation continue

- Association avec papetière

\section{Stratégie}

- Produit à plus de valeur ajoutée

- Fermeture

\subsection{Vision future du secteur et performance antérieure}

Les résultats montrent que selon le degré de succès des actions stratégiques antérieures, les décideurs perçoivent de façon significativement différente l'évolution future de leur secteur ainsi que de leur entreprise.

Au niveau sectoriel, les décideurs les plus performants entrevoient une restructuration importante au sein du secteur dontl'aboutissement sera une intégration plus poussée avec le secteur de pâtes et papier. De plus, ces décideurs perçoivent qu'il 
y aura une intensification de la recherche pour de nouveaux produits à plus grande valeur ajoutée et, par conséquent, une diversification accrue du secteur pour les années à venir. Notons que ce sont ici les mêmes décideurs qui avaient atteint une performance supérieure en misant sur une expansion de la capacité de fabrication de produits existants pour la période antérieure. Ceci suggère une évolution importante de leur cadre de vision stratégique depuis ce temps. La vision du profil plus performant se concentre de façon significative sur l'aspect marché pour lequel il entrevoit une évolution relativement stable des prix du bois d'oeuvre. Il est silencieux sur la dimension approvisionnement, alors que l'attention du profil moins performant porte de façon significative sur cet aspect. Ce dernier profil perçoit que l'implantation du nouveau régime forestier aura des impacts négatifs sur le secteur : coûts de matière première plus élevés, obligations de participation financière aux opérations de reboisement et exigence de plans de coupe précis requis par le ministère.

\subsection{Vision future de l'organisation et performance antérieure}

Les résultats de l'analyse discriminante montrent que la vision entretenue par les décideurs plus performants pour l'avenir de leur entreprise porte surtout sur les dimensions de marché et de stratégie, alors que pour le profil moins performant elle porte en plus sur la dimension approvisionnement. Ce qui distingue ces deux visions organisationnelles, ce sont surtout les caractéristiques particulières de chacune de ces dimensions. Du point de vue marché, la vision des plus performants se concentre sur l'impact à long terme d'une surtaxe à l'exportation aux Etats-Unis, dans l'éventualité où elle s'avèrerait de nature permanente. Cette attention est révélatrice de l'importance du marché américain pour ces entreprises. Pour le profil moins performant, la vision marché est tributaire de sa vision approvisionnement. Cette vision est caractérisée par une forte incertitude de pouvoir maintenir, même à un volume réduit, une continuité d'approvisionnement, ce qui engendre une incertitude quant aux perspectives des ventes futures.

Du point de vue des stratégies futures, c'est le profil plus performant qui se distingue surtout par ses choix de diversification de produits. Sur la dimension marché, la caractéristique du profil moins performant apparaît être l'acceptation des conséquences de sa contre-performance antérieure. C'est pourquoi les décideurs envisagent une diminution de leurs opérations ou encore recherchent une association avec une papetière, tout en maintenant un certain statu quo au niveau des orientations actuelles.

\section{Conclusion}

En conclusion, l'analyse de la vision stratégique tend à montrer qu'elle constitue un outil de gestion qui peut avoir deux types d'applications précises selon que l'on soit praticien ou chercheur. 
Pour le praticien, la vision représente un outil de gestion, un cadre qui anime et oriente ses décisions d'affaireset qui identifie sesopportunités d'avenir(Shrivastava et Mitroff, 1984). La vision stratégique donne une cohérence aux actions de l'entreprise. Elle permet 1) de définir avec force et clarté les buts et moyens futurs pour l'entreprise; 2) d'identifier et de réaliser des opportunités qui soient cohérentes avec ces objectifs; 3) de fournir au personnel de l'entreprise un cadre de référence cohérent et motivateur en vue d'orienter leur comportement individuel. Ces impacts gestionnels de la vision auront par le fait même une influence positive sur les activités de l'entreprise en ce qu'elles auront pour effet d'éliminer ou de prévenir les actions et décisions contradictoires ou confuses. Dans ce sens, la vision stratégique est un outil qui permet de gérer le lien entre les espoirs, les intentions, les valeurs, les traditions de l'entreprise et les activités quotidiennes des individus de l'entreprise. C'est donc un cadre qui, pour être efficace, doit être continuellement alimenté et renouvelé. En somme, une vision peut et devrait être gérée et entretenue. Pour ce faire, il est important de retenir qu'elle paraît être fortement influencée par les succès et les échecs antérieurs. En ce sens, il peut être nécessaire de la reconstruire de fond en comble suite à un échec entrepreneurial. Egalement, la vision devrait être approchée avec humilité et réalisme : elle ne constitue pas une vérité révélée. «La vision s'illusionne sur son propre compte lorsqu'elle se prend pour une vision, parce qu'elle est essentiellement un faire». (Castoriadis, 1973).

Pour le chercheur, l'étude de la vision stratégique permet d'établir un lien logique entre, d'une part, les actions du praticien et les décisions futures qu'il projette pour son entreprise et, d'autre part, entre ces actions, visions et les comportements culturels des individus dans l'entreprise. A un niveau sectoriel l'analyse de vision stratégique apparaît pouvoir avantageusement être combinée à l'approche microéconomique en vue d'établir un lien prospectif entre ces visions et actions et l'évolution future d'un secteur donné. Ceci pour en arriver à une meilleure prévision des orientations futures probables d'un secteur industriel. Cette approche pourrait s'avérer un outil de prospective particulièrement efficace pour prédire l'évolution de secteurs à forte densité de $P M E$, compte tenu que dans ces secteurs 1) la relation entre la vision d'un décideur dominant et ses actions stratégiques est plus directe et, également, compte tenu que 2) ce sont ces décideurs et les PME d'un secteur qui sont souvent en meilleure position de percevoir et de saisir les opportunités de nouvelles niches au sein d'un secteur. 


\section{BIBLIOGRAPHIE}

Bennis, W., (1982), «Leadership transforms vision into actions», Industry Week, mai, p. 5456.

Bennis, W. et Nanus, B., (1985), Leaders:The StrategiesforTaking Charge., New York, Harper \& Row.

Bird, B., (1988), «Implementing entrepreneurial ideas : the case for intention», Academy of Management Review, vol. 13, $\mathrm{n}^{\circ} 3$, p. 442-453.

Carrière, J.-B., (1990), «La décision stratégique d'innovation de procédé et la performance de l'organisation»., Thèse de doctorat (Ph.D.), Faculté des sciences de l'administration, Université Laval, Québec.

Castoriadis, C., (1973), La société bureaucratique, Union générale d'éditions, Collection 10/ 18.

Donaldson, G. et Lorsch, J.-W.,(1983), Decision Making at the Top :The Shaping of Strategic Decision., New York, Basic Books Inc..

Gupta, A.K., (1984), «Contingency linkages between stragegy and general manager characteristics : a conceptual examination», Academy of Management Review, 9, p. 399412.

Kirzner, I.-M., (1984), «The entrepreneurial process», dans C.A. Kent (éd.), The Environment for Entrepreneurship, Lexington Mass., D.C. Heath \& Co., p. 41-58.

Mendell, J.S. et Gerjuoy, H.G., (1984), «Anticipatory management or visionary leadership : a debate», Managerial Planning, 33, p. 28-31.

Moscovici, S., (1981), «On social representations», dans J.P. Forgas (éd.), Social Cognition : Perspectives on Everyday Understanding, London, Academic Press.

Quinn, J.B., (1982), «Managing strategies incrementally», Omega, vol. 10, n 6, p. 613-627.

Searle, J., (1984), Minds, Brains and Science. Cambridge Mass., Harvard University Press.

Seldon, A., (1984), «Preface» dans C.A. Kent (éd.). The Environment for Entrepreneurship. Lexington Mass., D.C. Heath \& Co., p. I - XX.

Shrivastava, P. et Mitroff, I.-I., (1984), «Enhancing organizational research utilization : the role of decision-maker's assumptions», Academy of Management Review, vol. 9, $\mathrm{n}^{\circ} 1, \mathrm{p}$. 18-26.

Simon, H.A., (1986), «What we know about the creative process», dans R.L. Kuhn (éd.), Frontiers in Creative and Innovative Management, Cambridge Mass., Ballinger Publishing Co., p. 3-21.

Westley, F. et Mintzberg, H., (1989), «Visionary leadership and strategic management», Strategic Management Journal, 10, p 17-32. 